Sitientibus Série Ciências Físicas 03: 18-31 (2007)

\title{
Imagens Magnéticas para Aplicações Biomédicas: estado da arte
}

\author{
Magnetic Images for Biomedical Applications: state of the art
}

\author{
Juan A. Leyva Cruz* e Thierry J. Lemaire ${ }^{\dagger}$ \\ Laboratório de Instrumentação em Física, Departamento de Física - UEFS \\ Campus Universitário Km 03 BR 116, Feira de Santana-BA, 44031-460
}

\begin{abstract}
Na atualidade, o estudo da energia eletromagnética aplicada à medicina e biologia é um dos tópicos de pesquisas mais atraentes e de interesse crescente na comunidade científica internacional, e particularmente no Brasil. Vários esforços têm sido realizados para criar técnicas diagnósticas apoiadas na obtenção de imagens magnéticas e elétricas para ajudar de forma coadjuvante, e/ou substituir em alguns casos, as metodologias médicas de diagnóstico por imagens tradicionais. Neste trabalho mostramos o estado da arte, do ponto de vista experimental, destacando as mudanças nas dimensões dos sensores e no sistema de condicionamento analógico dos sinais medidos, para o registro de sinais de origem biológica. A imagem magnética medida sempre será degradada e ruidosa, devido tanto ao princípio físico (Lei Biot-Savart) envolvido na formação da imagem quanto ao próprio processo de medição da imagem. Os fundamentos básicos da formação das imagens magnéticas, bem como a solução do problema magnético direto e inverso, são mostrados. Apresentamos uma discussão do ponto de vista físico-matemático sobre o problema magnético inverso mal-condicionado e como ele influi na reconstrução das imagens das fontes magnéticas no interior de um objeto qualquer. Por fim, mencionamos de forma breve o método de filtragem espacial de Fourier como uma das possíveis técnicas na solução do problema magnético inverso.
\end{abstract}

Palavras-chaves: biomagnetismo, imagens magnéticas, bioinstrumentação, sensores magnéticos, problema inverso.

At the present, the study of electromagnetic energy applied in Medicine and Biology is one of the most attractive topics of research and one of growing interest in the international scientific community, as well as in Brazil. Many efforts have been done to established diagnose techniques supported in taking magnetic and electric images for helping as an adjuvant way, or in some cases replacing, the medical methodologies on diagnosis by traditional images. In this work, we shows the state of art, from an experimental point of view, highlighting the changes in sensor dimensions and in the analogical conditioning system of the measured signs, to record signs of biological origin. The measured magnetic image always be degraded and noised, due to the physical principle (Biot-Savart Law) involved in the image formation as to the very process of measurement of image. The basic fundamentals in the formation of magnetic images, as well the solution for the direct and inverse magnetic problem, are showed. We present a discussion, from a mathematical-physical point of view about the ilconditioned inverse magnetic problem and how it affects the image reconstruction of magnetic sources within any object. Finally, we mention briefly the Fourier spatial filtering method as one of possible techniques for the solution of inverse magnetic problem.

Key-words: biomagnetism, magnetic images, bioinstrumentation, magnetic sensors, inverse problem.

\section{INTRODUÇÃO}

*Endereço Eletrônico: jalbertoleyva@yahoo.com.br

${ }^{\dagger}$ Endereço Eletrônico: tj_lemaire@yahoo.com.br

Campos biomagnéticos ou campos magnéticos biológicos têm sua origem nas correntes elétricas que percorrem as células 
(como no sistema nervoso e no coração) ou em materiais magnéticos acumulados em certos órgãos (estômago, fígado e pulmão) [1-5]. Estudos experimentais $[6,7]$ têm mostrado que os campos magnéticos produzidos pelo corpo humano e por outros seres são extremamente tênues, na faixa de 100 a 20 [6-10]. Medir tais campos e resolver o problema inverso associado nos permite localizar com precisão a região que os produz e determinar a intensidade da corrente ou a concentração das partículas magnéticas que os originaram. Essa tarefa é dificultada por sua baixa intensidade e pela presença de outros campos magnéticos (da Terra e da rede elétrica) muito mais intensos e chamados de ruído magnético ambiental. Para registrar estes sinais biogênicos precisa-se de equipamentos muito sensíveis tais como, magnetômetros supercondutores de interferência quântica ou SQUIDS (siglas de Superconducting Quantum Interference Device), os quais são magnetômetros muito custosos e de difícil manutenção. Fato este, que faz sua aplicação clínica muito limitada. O procedimento que estuda a obtenção e análises da distribuição espacial e temporal dos campos biomagnéticos e de suas fontes é chamado de Técnica de Imagens Biomagnéticas. Exemplos deste tipo de imagens são; Magnetoencefalografia (MEG), Magnetocardiografia (MCG) e Magnetogastrografia (MGG), por meio das quais se podem estudar a fisiologia do cérebro, coração e da região gástrica [11].

Os estudos biomagnéticos tipicamente utilizam sensores SQUIDS com diâmetros da ordem de $(10-30) \mathrm{mm}$, magnitude aproximadamente igual à distância entre estes sensores e a amostra biológica. Apesar das medidas de tais sistemas serem usadas para localizar fontes magnéticas dipolo-dipolo com exatidão na casa de alguns milímetros (>2 mm), a capacidade de resolver ou discriminar dois dipolos adjacentes num raio de dois $\mathrm{mm}$ ou menores, fica limitada pelas dimensões dos sensores e das distâncias sensores-fontes, como no caso das imagens obtidas na MCG e MEG.

Apesar da resolução obtida por tais sistemas ser suficientemente útil para diversos estudos das doenças no homem, a mesma apresenta-se inadequada para estudos a nível celular. Um exemplo é a corrente de ação na despolarização e repolarização das células cardíacas, as quais acontecem na escala de centenas de micrometros até $1 \mathrm{~mm}$. Neste caso, uma boa caracterização exige a obtenção de imagens magnéticas de alta resolução, ou seja, na mesma escala espacial. Vários trabalhos têm demonstrado os benefícios da obtenção de imagens de alta resolução e têm mostrado as bases para entender os fatores que governam a resolução espacial das mesmas. É importante realçar, que não somente o uso de fatores simples como o da escala têm influenciado o aumento ou melhora deste tipo de sistemas de imagens, o que está governado pela sensibilidade dos sensores. Hoje se sabe que dito desempenho é modificado pelo grau de espalhamento no espaço tridimensional $(3 D)$ do campo produzido pelas fontes magnéticas distribuídas bidimensionalmente $2 D$ ou $3 D$. Por exemplo, investigações anteriores têm mostrado que a técnica de MCG multicanal (MCGM) pode ser usada para obter-se imagens biomagnéticas cardíacas funcionais de forma não-invasivas, as quais facilitam entre outras a localização de arritmias, de modo que o número de hospitais onde a MCGM está instalada está crescendo [12, 13].

Na Figura 1, ilustramos os resultados de um estudo experimental no qual foram obtidas as imagens da distribuição do potencial de membrana (PM) e do campo biomagnético bidimensional (neste caso usando SQUIDs) geradas pelas correntes elétricas que circulam nas células do coração isolado de um coelho (ver fotografia na Figura 1, parte (a)), após estímulos elétricos usados para reativar seu funcionamento [14]. O objetivo deste estudo foi aumentar a sensibilidade dos sensores magnéticos do tipo SQUIDs, modificando suas dimensões de $(10-30) \mathrm{mm}$ para $2 \mathrm{~mm}$, e introduzindo novas formas de processamento dos sinais multilaços de correntes com retroalimentação. $\mathrm{Na}$ Figura 1 (parte (b)) apresentam-se segmentos das formas das ondas dos sinais do PM e do campo biomagnético medidos no mesmo local sobre a superfície do coração. Na Figura 1 (parte (c)) vemos a imagem da distribuição do PM $53 \mathrm{~ms}$ depois da estimulação elétrica 
do coração. As regiões mais avermelhadas significam regiões de despolarização $(V / V \max \sim$ 1 ), e as azuis representam regiões predominantes pelo potencial de repouso $\left(V / V_{\max } \sim 0\right)$. Nas imagens podemos identificar facilmente as frentes de ondas elétricas cardíacas, que são regiões fronteiriças entre as regiões de despolarização e de repouso do PM. Como se podem observar na imagem da Figura 1, duas frentes de ondas originadas posteriormente ao estímulo estão prestes a colidir [14]. Este trabalho demonstra que modificando as dimensões dos sensores magnéticos, entre outros fatores, se pode conseguir estudar de forma mais detalhada a relação entre PM extracelulares, o campo biomagnético cardíaco e as correntes de ação celular que os geram, especificamente em camadas de tecido cardíaco conectivo. Logrando-se verificar processos em escalas mais próximas daquelas que acontecem os processos fisiológicos in vivo, que neste caso é da ordem de $1 \mathrm{~mm}$.
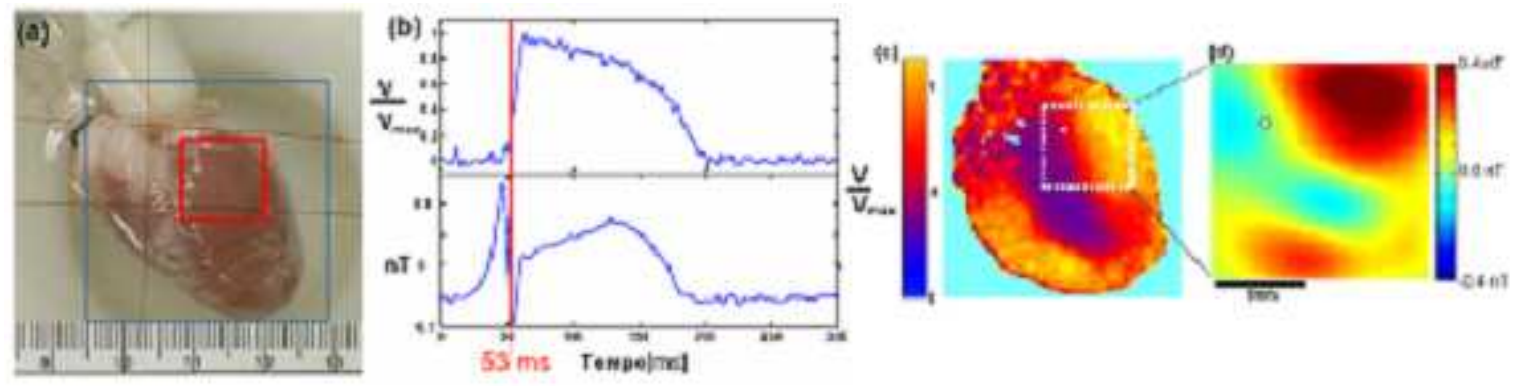

FIGURA 1: (a) Imagem fotográfica mostrando as áreas do coração de um coelho usado para medidas do potencial de membrana $(\mathbf{P M})$ das células cardíacas (quadrado azul) e o campo magnético (quadrado vermelho). (b) Forma das ondas dos sinais PM e do campo biomagnético medido no mesmo local sobre a superfície do coração. (c) Imagem da distribuição do PM 53 ms após o estímulo elétrico. Nela vemos duas frentes de ondas de lados opostos prestes a colidir. (d) Imagem da indução magnética também $53 \mathrm{~ms}$ depois, desde a área indicada pelo quadrado branco mostrado em (c). Extraída da referência [14].

Os campos biomagnéticos originados pelo sistema gástrico também são alvos de numerosos estudos. Uma boa parte dos alimentos digeridos é absorvida devido ao processos bioquímicos e biofísicos. Ambos processos produzem movimentos mecânicos ou peristálticos. A causa destes últimos é a despolarização das membranas das células dos músculos lisos do estômago, a qual origina uma atividade elétrica nesse órgão, semelhante a um marca-passo situado no corpo do mesmo, que se converte numa onda mecânica que avança desde o corpo até o antro, com um tempo de duração de aproximadamente $20 \mathrm{~s}$. Isto faz com que apareça um padrão de frequência característico da atividade elétrica neste órgão, sendo a mesma da ordem de $0,05 \mathrm{~Hz}$ ou três ciclos por minuto $[5,15,16]$. Esta atividade mecânica é conhecida como motilidade gástrica.

Dentro da área do gastromagnetismo, além de estudar-se os campos biomagnéticos natu- rais ou MGG, as pesquisas relacionadas com a detecção dos campos biomagnéticos produzidos por partículas magnéticas ou ímãs ingeridos e magnetizados previamente têm chamado a atenção da comunidade científica mundial, e especialmente no Brasil [17-21]. Devido a essa nova frente de pesquisa, muitos agentes de contrastes transportados vêm sendo desenvolvidos e desenhados para se ligar ou se incorporar em dados tipos de células ou órgãos, envolvidos em algum processo de uma determinada doença. Estes agentes transportam quantidades de marcadores ou segmentos de moléculas, que servem para dar melhor contraste às imagens ou mesmo produzí-las. Neste caso estes marcadores são micropartículas ferrimagnéticas ou superparamagnéticas e nanopartículas magnéticas (Ferridex). Quando se transportam ímãs, estes transportadores são denominados marcadores magnéticos (MM) [22] e quando são utilizadas 
partículas em suspensão ou dispersas, são chamados traçadores magnéticos (TM) [23]. Estes materiais são introduzidos propositalmente no interior da região gastrintestinal ou cardíaca para dotá-la, de forma artificial, de uma capacidade de gerar campos magnéticos locais mais intensos que os biogênicos. Consequentemente, pode-se detectá-los de forma simples e não-invasiva através de medições na superfície do estômago e torso, utilizando sensores magnéticos não tão custosos quanto os SQUIDs, como os magnetômetros fluxgates, biosusceptômetros de corrente alterna (BCA) e os sensores magnetoresistivos (MR).

Em estudos prévios têm-se demonstrado que estes tipos de contraste, uma vez magnetizados, produzem campos magnéticos que podem afetar até os sinais da ressonância magnética nuclear [24]. Nestas condições é possível medir tais campos por magnetômetros de alta sensibilidade, não convencionais, à temperatura ambiente. Trabalhos realizados pelo grupo de pesquisa em Biomagnetismo de Ribeirão Preto, da Universidade de São Paulo, têm estudado a motilidade gástrica usando traçadores magnéticos, os quais podem ser ingeridos em conjunto com uma refeição teste, e posteriormente magnetizando as partículas magnéticas com um campo magnético intenso, da ordem de $80 \mathrm{mT}$. Inicialmente o pó de ferrita de manganês misturado com iogurte foi usado como TM para medir o tempo de esvaziamento gástrico usando um BCA, no qual se detectou o campo magnético remanente gerado por uma concentração do ferrita de até 3\% [25]. Moreira et al. (2001) [26] realizaram estudos relacionados com a obtenção das imagens das fontes magnéticas de TM usando um sistema baseado num BCA. As imagens foram obtidas resolvendo o problema magnético inverso, utilizando o método da filtragem espacial de Wiener paramétrica, generalizada, bidimensional.

Todos os trabalhos anteriores foram realizados utilizando-se somente um sensor magnético para a detecção monocanal num ponto fora do corpo. Levando em conta o anterior, foi que se passou a estudar o uso de sistemas multicanais de medição de campos magnéticos usando diferentes arranjos e tipos de sensores, para assim obter maior volume de informação em vários pontos. Consequentemente, criando as condições práticas em termos da informação suficiente para obtermos uma imagem magnética com alguma utilidade clínica.

Recentemente, Cano et al. (2005) [27] publicaram os resultados da construção de um sistema de imagens magnéticas usando um arranjo linear de sensores magnetorresistivos. Nesse estudo os autores reportam a obtenção de imagens magnéticas com excelente resolução espacial. Por outro lado, Corá et al. (2006) [28],têm empregado a mesma técnica multicanal com vários BCA, para avaliar por meio de imagens biomagnéticas, o processo de desintegração de drogas ligadas à partículas magnéticas.

Cruz et al. (2006) [29] reportam a construção de um sistema multicanal automático de imagens biomagnéticas (SMIB) utilizando um arranjo multicanal com 12 sensores magnetoresistivos do tipo HMC-1001/1002. Segundo os autores, o SMIB realiza a varredura num tempo menor que um minuto numa área de $18 \times 16 \mathrm{~cm}^{2}$, e executa medições simultâneas dos 12 detectores magnéticos. Nesse trabalho foi criada uma interface computacional escrita na linguagem computacional $M A T L A B^{\circledR}$ que foi utilizada para obtenção das imagens magnéticas bidimensionais e no cálculo das imagens das fontes magnéticas para diferentes fantomas magnéticos em $2 D$. As imagens reconstruídas das fontes magnéticas foram obtidas utilizando vários paradigmas de solução do problema magnético inverso. As imagens foram reconstruídas usando-se a função de espalhamento pontual experimental e teórica. Em ambos os casos, os procedimentos de reconstrução fazeram uso das imagens magnéticas experimentais para uma distância sensor-fonte.

Segundo os autores, as imagens reconstruídas mostraram uma melhora na resolução espacial quando foram obtidas usando-se a função de espalhamento teórica do SMIB. Usando-se este sistema de imagens foi demonstrada a capacidade de produzir imagens magnéticas da ordem de poucos $n T$, utilizando-se um arranjo de sensores magnetoresistivos do tipo HMC 
1001/1002, com uma densidade espectral de ruído da ordem de $150 \mathrm{pT} \cdot \mathrm{Hz}^{-0.5}$ por sensor [30]. Nesse trabalho mostramos que a técnica de imagens magnéticas exibe perspectivas promissoras nas aplicações biomédicas a curto prazo.

\section{FORMAÇÃO DE UMA IMAGEM MAGNÉTICA BIDIMENSIONAL (2D)}

Vamos considerar um modelo físico formado por uma região do espaço, $\Omega$, onde se cumpra a métrica de Hilbert, e no qual posicionamos um corpo/objeto ou amostra (C) de espessura muito pequena e igual a $\epsilon$, formado por partículas de magnetita $\left(\mathrm{Fe}_{3} \mathrm{O}_{4}\right)$ não tratada termicamente e com uma concentração $C_{\mathrm{Fe}_{3} \mathrm{O}_{4}}\left(x^{\prime}, y^{\prime}\right)$, gerando um vetor momento magnético $\vec{m}\left(x^{\prime}, y^{\prime}\right)$ em duas dimensões, caracterizando a magnetização da amostra, e que produz um campo de indução magnética $\vec{B}\left(x, y, z_{0}\right)$ a uma distância $z_{0}$ acima da amostra, e apenas na direção- $z$. Esta última condição é garantida magnetizando a amostra nessa direção. Do ponto de vista do Bioeletromagnetismo tal objeto é chamado de volume condutor bioelétrico. Como podemos observar na Figura 2, o problema de obter uma imagem magnética passa por três elementos ou etapas fundamentais a saber:

1- O objeto,

2- Sistema de medições,

3- Formação da imagem magnética.

Ao mesmo tempo essas três partes definem o espaço ou plano objeto (PO), das medições $(\mathrm{PM})$ e a imagem $(\mathrm{PI})$, respectivamente.

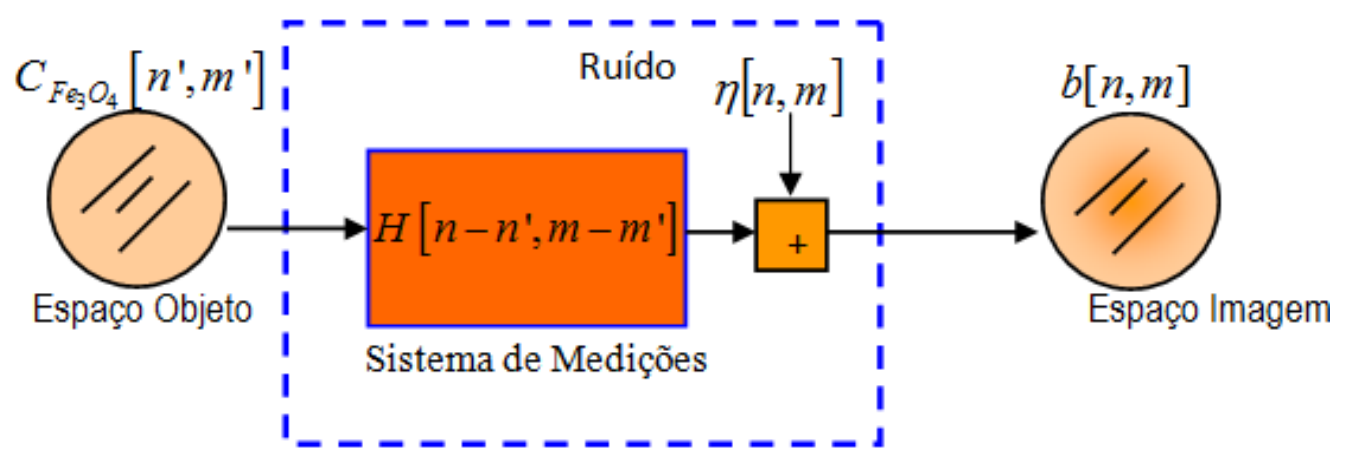

FIGURA 2: Esquema do processo de formação de uma imagem magnética degradada, $b_{d}(x, y, z-\epsilon)$, a qual é influenciada pelo operador degradador e pelo sistema de medições que a contaminam com ruídos $\eta(x, y)$.

Na Figura 2 mostramos um diagrama geral que explica o processo de formação de uma imagem magnética, que relaciona o $\mathrm{PO}$ representando o objeto-C, no interior do qual se encontra a distribuição espacial da magnetização, propriedade física da qual queremos obter sua imagem real, o PM representa o sistema que realiza as medições do campo magnético nos pontos de uma rede finita acima da amostra (também conhecido como processo de discretização), e o PI é aonde se realiza a formação ou quantização da imagem magnética detectada, neste processo geralmente se faz uma codificação entre os valores medidos e um dado mapa de cores falso. A imagem magnética transporta informação das propriedades físicas do objeto (magnetização, dentre outras) as quais não são diretamente observáveis. Em geral a informação consiste numa representação degradada de uma dada grandeza física gerada pelo objeto original em função do espaço, em duas ou mais dimensões incluindo o tempo. Neste processo são destacados dois eventos principais que produzem a degradação da imagem magnética. O primeiro é o processo físico de formação da imagem e o segundo o processo 
de registro ou detecção da imagem, pelo sistema de medição de imagens.

A imagem magnética degradada ou distorcida bidimensional, $b_{d}(x, y, z-\epsilon)$, é o resultado da resposta do sistema, $h\left(x-x^{\prime}, y-y^{\prime}, z-\epsilon\right)$, modelada por meio do operador degradador, considerado linear e invariante a deslocamentos espaciais atuando sobre a imagem do momento magnético bidimensional (imagem das fontes), $\vec{m}_{\mathrm{Fe}_{3} \mathrm{O}_{4}}\left(x^{\prime}, y^{\prime}\right)$, da amostra de espessura $\epsilon$, como sugere o diagrama lógico da Figura 2, processo que pode ser representado pela equação integral de Fredholm de primeira espécie:

$$
\begin{aligned}
b_{d}(x, y, z-\epsilon) & =\int_{x^{\prime}} \int_{y^{\prime}} h\left(x-x^{\prime}, y-y^{\prime}, z-\epsilon\right) \times \\
& \times \vec{m}_{\mathrm{Fe}_{3} O_{4}}\left(x^{\prime}, y^{\prime}\right) \epsilon d x^{\prime} d y^{\prime} \\
& +\eta(x, y, z-\epsilon),
\end{aligned}
$$

aqui $h\left(x-x^{\prime}, y-y^{\prime}, z-\epsilon\right)$ também representa a função impulsiva do sistema ou função de espalhamento pontual,PSF,(PSF, das siglas em inglês Point Spread Function). Precisamente o efeito da PSF é o do operador degradador que produz a degradação da imagem devido ao processo físico de formação da imagem, o qual é considerado como determinístico. $\mathrm{O}$ último termo, $\eta(x, y, z-\epsilon)$, é o ruído independente dentro do qual consideramos os erros experimentais, o ruído magnético ambiental, ruído de interferências de radiofrequências e da rede, ruídos introduzidos pelos componentes eletrônicos do sistema de imagens, dentre outros, os quais contaminam a imagem de ruído estatístico durante o processo de registro experimental da imagem magnética. Este ruído é em geral um processo aleatório e na prática é desconhecido. O que pode ser conhecido sobre o ruído são as suas propriedades estatísticas tais como: o valor médio, variância, se é de tipo aditivo ou multiplicativo, correlacionado ou não-correlacionado, se segue a distribuição Gaussiana ou Poisson, dentre outras, que quando conhecidas a priori podem ser utilizadas para o tratamento do problema. A quantidade de ruído presente numa imagem magnética degradada discreta pode ser medida por meio da chamada razão sinal-ruído, SNR,
(SNR, das siglas em inglês noise-to-signal ratio) a qual pode ser definida por

$S N R=10 \log \left[\frac{\text { variação do sinal de interesse }}{\text { variação do ruído }}\right]$

enquanto que, em termos práticos, o valor da $S N R$ determina-se por

$$
S N R=20 \log \left[\frac{\left\|b_{d}(x, y, z-\epsilon)\right\|}{\|\eta(x, y, z-\epsilon)\|}\right]
$$

onde as normas euclidianas estão associadas ao produto escalar. Quando a SNR do sistema de imagens é da ordem (40 a 50) dB, o nível de ruído não é visível na imagem magnética degradada e os efeitos causados pelo operador degradador são dominantes.

Na prática os objetos são finitos e as funções que neles são definidas são espaços-limitados, e porem as imagens magnéticas experimentais também o é. Estas últimas são obtidas, por médio da amostragem dos dados do campo magnético, num arranjo ou rede de $(m \times n)$ pontos de detecção, acima da amostra discretizada. Portanto, o modelo da Eq. (1) pode ser escrito como:

$$
\begin{aligned}
& b_{d}\left(x_{n}, y_{m}, z-\epsilon\right) \\
= & \sum_{n^{\prime}=0}^{n-1} \sum_{n^{\prime}=0}^{n-1} h\left(x_{n}-x_{n}^{\prime}, y_{m}-y_{m}^{\prime}, z-\epsilon\right) \times \\
\times & \vec{m}_{F_{3} O_{4}}\left(x_{n}^{\prime}, y_{m}^{\prime}\right)+\eta\left(x_{n}, y_{m}, z-\epsilon\right),
\end{aligned}
$$

onde $n^{\prime}$ e $m^{\prime}$ representam os índices da discretização da amostra nas direções $x$ e $y$, respectivamente.

A formação da imagem magnética degradada em notação matriz-vetor desde a Eq. (3) pode ser expressa matematicamente por:

$$
\begin{aligned}
& b_{d}\left(x_{n}, y_{m}, z-\epsilon\right) \\
= & \sum_{n^{\prime}=0}^{n-1} \sum_{n^{\prime}=0}^{n-1} h\left(x_{n}-x_{n}^{\prime}, y_{m}-y_{m}^{\prime}, z-\epsilon\right) \times \\
\times & \vec{m}_{F e_{3} O_{4}}\left(x_{n}^{\prime}, y_{m}^{\prime}\right)+\eta\left(x_{n}, y_{m}, z-\epsilon\right),
\end{aligned}
$$

A expressão anterior representa a convolução 
entre a função de resposta impulsiva do sistema e a função que representa o objeto fonte. O problema de reconstrução de imagens é encontrar uma estimativa da imagem das fontes ou da distribuição do vetor momento magnético $\vec{m}_{\mathrm{Fe}_{3} \mathrm{O}_{4}}\left(x_{n}^{\prime}, y_{m}^{\prime}\right)$ conhecendo-se a imagem magnética degradada $b_{d}\left(x_{n}, y_{m}, z-\epsilon\right) \mathrm{e}$ a função impulsiva $h\left(x_{n}-x_{n}^{\prime}, y_{m}-y_{m}^{\prime}, z-\epsilon\right)$, do sistema linear de imagens. Isto não é um problema trivial porque mesmo se o operador é conhecido, uma solução trivial ou ingênua (naive termo usado em inglês) poderia amplificar o conteúdo do ruído, ou produzir uma não-solução do problema inverso, como veremos mais adiante.

O problema na obtenção da distribuição espacial das propriedades eletromagnéticas no interior de um objeto volume condutor (por exemplo, o corpo humano) vem crescendo nos últimos anos. Medições experimentais ou simulações teóricas da distribuição espacial da intensidade da indução do campo magnético produzida por qualquer fonte magnética é o mesmo que a obtenção de mapas ou imagens magnéticas em $2 D$ ou $3 D$. Esta teoria matemática aplicada ao Bioeletromagnetismo é conhecida como problema bioelétrico/magnético direto (PMD). Ao contrário, o processo de obtenção das imagens das propriedades das fontes magnéticas desconhecidas, partindo dos mapas magnéticos e outras informações a priori, é chamado de problema bioelétrico/magnético inverso (PMI).

\section{PROBLEMA MAGNÉTICO INVERSO MAL-CONDICIONADO}

Desde o ponto de vista fenomenológico, o problema inverso pode ser descrito como o procedimento lógico dado pela sequência: efeito $\rightarrow$ sistema $\rightarrow$ causa. Em nosso casso, o PMI no volume condutor bioelétrico, seria obter as imagens das fontes magnéticas, ou seja, do momento magnético ou da magnetização, as quais representariam as "causas", partindo do conhecimento da PSF do sistema de imagens, da imagem magnética degradada e ruidosa medida experimentalmente, que é o "efeito", alem de outras informações a priori, estas normalmente de natureza estatística. A solução de um problema deste tipo seja na medicina ou qualquer outra área de aplicação, consiste em determinar as causas baseado na observação dos seus efeitos.

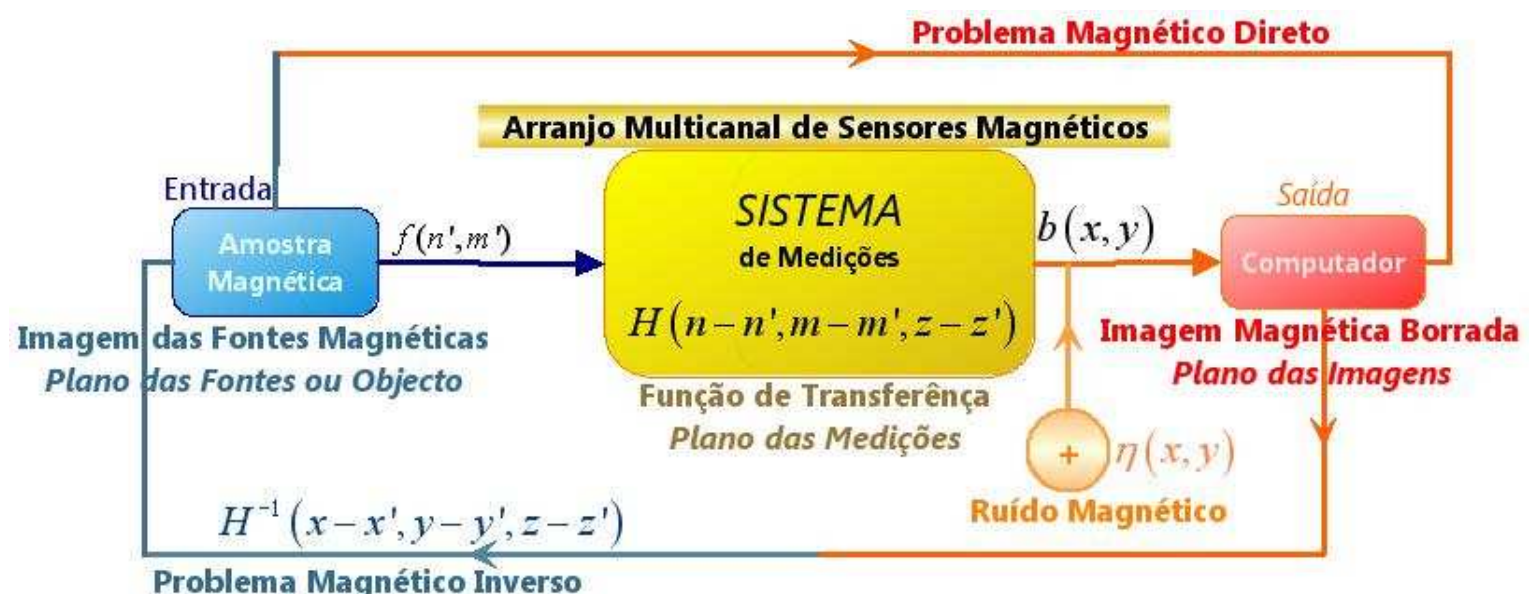

FIGURA 3: Diagrama mostrando o sistema de imagens, modelado por um sistema linear invariante no espaço-tempo. Vê-se a entrada $\rightarrow$ sistema $\rightarrow$ saída, queexplica a formação de uma imagen magnética.

Ao mesmo tempo o processo de formação da imagem magnética degradada experimental pode ser pensado como sendo um filtro digi- tal que computa uma sequência ou sinal discreto na sua saída filtrando uma sequência ou sinal de entrada também discreta. Em geral 
um modelo físico que descreva a formação da imagem magnética e consequentemente, o problema magnético inverso consiste num modelo matemático conceitualmente simples: um sistema de equações lineares algébricas. $\mathrm{O}$ esquema deste modelo é mostrado na Fig. (3), o sistema deve ser linear, invariante no espaçotempo e relacionar os sinais de entrada $\rightarrow$ com o sistema de medições $\rightarrow$ e os sinais de saída do sistema linear de imagens. Assim, aplicando o teorema da convolução o qual garante que ao computar as Transformadas de Fourier ao modelo descrito pela Eq. (4), poderemos descrevêlo no domínio das frequências espaciais, como um sistema de equações lineares da seguinte forma matricial,

$$
\begin{aligned}
& H\left[K_{x}-K_{x^{\prime}}, K_{y}-K_{y^{\prime}}\right] M_{F_{3} O_{4}}\left[K_{x^{\prime}}, K_{y^{\prime}}\right] \\
= & B_{z}\left[K_{x}, K_{y}\right]
\end{aligned}
$$

sendo $\left[K_{x^{\prime}}, K_{y^{\prime}}\right]$ as coordenadas da frequência espacial nas direções x e y, respectivamente. Os termos $M_{\mathrm{Fe}_{3} \mathrm{O}_{4}}\left[K_{x^{\prime}}, K_{y^{\prime}}\right]$ e $B_{z}\left[K_{x}, K_{y}\right]$ são as imagens das fontes magnéticas e da imagem magnética degradada na direção $-z$, no domínio das frequências espaciais, sendo que nesta última se encontra embutido o aporte do ruído aditivo. O termo $H\left[K_{x}-K_{x^{\prime}}, K_{y}-K_{y^{\prime}}\right]$ é a transformada de Fourier bidimensional da PSF do sistema. Também é conhecida como Função de Transferência Ótica-OTF (siglas da frase em inglês Optical Transfer Function) e o módulo desta última é chamado de Função de Transferência de Modulação-MTF (das siglas em inglês Modulation Transfer Function) muitas vezes correspondem a matrizes retangulares as quais são estruturas matemáticas típicas de muitos problemas inversos. Estas funções são os operadores degradador que caracterizam a qualidade do sistema linear de imagens magnéticas em obter imagens magnéticas com boa ou não, resolução espacial e temporal.

O fato de poder escrever a Eq.

no "domínio de Fourier" traz uma grande vantagem, porque se a matriz $H\left[K_{x}-K_{x^{\prime}}, K_{y}-K_{y^{\prime}}\right]$ possui uma inversa (normalmente existem inversas para as matrizes quadradas) dada por
$H^{-1}\left[K_{x}-K_{x^{\prime}}, K_{y}-K_{y^{\prime}}\right]$ então nesse caso a solução do sistema é única e pode ser obtida ao multiplicarmos ambas as partes da Eq. (5) por $H^{-1}\left[K_{x}-K_{x^{\prime}}, K_{y}-K_{y^{\prime}}\right]$, chegando a,

$$
\begin{aligned}
& H^{-1}\left[K_{x}-K_{x^{\prime}}, K_{y}-K_{y^{\prime}}\right] B_{z}\left[K_{x}, K_{y}\right] \\
= & H^{-1}\left[K_{x}-K_{x^{\prime}}, K_{y}-K_{y^{\prime}}\right] \times \\
\times & \left(H\left[K_{x}-K_{x^{\prime}}, K_{y}-K_{y^{\prime}}\right] M_{F e_{3} O_{4}}\left[K_{x^{\prime}}, K_{y^{\prime}}\right]\right),
\end{aligned}
$$

obtendo finalmente,

$$
\begin{aligned}
& H^{-1}\left[K_{x}-K_{x^{\prime}}, K_{y}-K_{y^{\prime}}\right] B_{d}\left[K_{x}, K_{y}\right] \\
= & M_{\mathrm{Fe}_{3} \mathrm{O}_{4}}\left[K_{x^{\prime}}, K_{y^{\prime}}\right]
\end{aligned}
$$

A qual representa a solução do problema magnético inverso no domínio de Fourier. Em conexão com a teoria de problemas inversos, a solução dada em pela Eq. (7) é chamada de solução simples ou "ingênua". A função $H^{-1}\left[K_{x}-K_{x^{\prime}}, K_{y}-K_{y^{\prime}}\right]$ é conhecida como filtro inverso e é o recíproco da resposta em frequência do sistema linear de imagens. $\mathrm{Na}$ prática o uso deste filtro não é o procedimento mais adequado, pois ele é muito sensível ao conteúdo do ruído aditivo presente e pode apresentar instabilidades entorno de pontos singulares (pontos aonde o filtro pode valer zero) que possam aparecer no plano de Fourier, provocando amplificação do ruído consequentemente uma solução dominada pelo ruído fazendo com que a imagem restaurada seja muito diferente da real. Por outro lado, se a matriz é retangular, então pode haver tanto infinitas como nenhuma solução sequer. Estudos [31-34] têm demonstrado que esta solução não é única, e a solução computada apresenta um grande erro, pelo que sua aplicação não é a melhor escolha, em comparação com os algoritmos baseados no controle do conteúdo de frequências do filtro inverso e porem o nível do ruído, tais como os métodos de regularização, dentre outros.

A solução ingênua não é boa estimativa da solução num PMI, isto é causado por várias razões, dentro das quais podemos citar a influência do ruído nas medições, diferentes causas poderiam produzir os mesmo efeitos, é principalmente o fato dos dados serem incompletos (não temos acesso a todas as informações 
disponíveis). Um exemplo da manifestação desta última e no caso da solução do problema bioelétrico inverso em eletrocardiografia, na qual sabemos que não podemos ter eletrodos para medir o ECG em todos os pontos do corpo, portanto, os dados medidos a princípio são incompletos. Em geral a solução do PMI é não-única e instável. Esta característica indesejada faz com que os problemas inversos matematicamente sejam chamados de problemas inversos mal-postos ou mal-condicionados (ill-posed/conditional: significado em inglês). Este tipo de problema não satisfaz as condições impostas em 1902, pelo matemático francês Hadamard [35] o qual publicou os postulados que deveriam ser satisfeitos para um problema matemático corresponder à realidade ou ser considerado bem-posto, os quais são: (a) a solução deve existir (existência); (b) a solução deve ser determinada pelos dados de forma única (unicidade) e (c) a solução deve depender dos dados de forma contínua (estabilidade).

Uma consequência da instabilidade de um problema mal-posto (solução não-existe) é que pequenas alterações nos dados medidos (aqui entra a influência do ruído, o qual assegura na prática esta condição) induz perturbações grandes na solução. Quando discretizamos um problema mal-posto, normalmente obtemos um problema bem posto (solução ingênua), com solução única a qual depende continuamente dos dados. Mais como foi visto está solução desde o ponto de vista físico não é aceitável, porque está completamente dominada pelo ruído.

Um indicador da condição (solução) do problema é o número da condição (nc), os problemas mal-postos ou mal-condicionados apresentam um número de condição extremamente grande, e implica em instabilidade numérica do filtro inverso. Por exemplo, um valor do $n c=106$, implica que um erro relativo na data da ordem de 10-6 poderia implicar num erro de $100 \%$ na solução. Um problema é definido bemcondicionado quando seu número de condição está perto de 1 . O nc é extremamente importante, pois mede a propagação do erro, ruído, desde os dados para a solução do problema inverso.
Em geral estes problemas são malcondicionados, pois somente numa situação idealizada é que podem se medir sinais ou dados exatos (sem ruído). Consequentemente a influencia do ruído é um problema muito serio a ter presente na hora de computar a solução destes tipos de problemas. Precisamente os métodos de regularização usados para a solução do PMI utilizam conhecimento a priori sobre o modelo físico e apóiam-se nessa condição para processar os dados medidos controlando o conteúdo do ruído aditivo presente, e assim para regularizar o problema, ou seja, converter o problema mal-posto em um bem-condicionado e obter uma solução plausível do problema magnético inverso.

\section{SOLUÇÃO DO PMI VIA FILTROS ESPACIAIS DE FOURIER}

O paradigma baseado nos filtros espaciais de Fourier aparece da aplicação direta das técnicas das frequências espaciais ou aplicação das transformadas de Fourier nas equações que modelam o processo de formação das imagens magnéticas e na obtenção das imagens das fontes magnéticas. Para obter uma solução do PMI simplificada usando estes filtros vamos usar o modelo do volume condutor bioelétrico descrito na seção 3 , considerando as fontes magnéticas em duas dimensões. Nestas condições o volume condutor num plano de coordenadas produz um campo magnético perpendicular nos pontos, que segundo a lei de Biot-Savart vem dado por

$$
\begin{aligned}
& d b_{z}(x, y, z-\epsilon) \\
& =\frac{\mu_{0}}{4 \pi} \frac{2\left(z-z^{\prime}\right)^{2}-\left(x-x^{\prime}\right)^{2}-\left(y-y^{\prime}\right)^{2}}{\left[\left(x-x^{\prime}\right)^{2}+\left(y-y^{\prime}\right)^{2}+(z-\epsilon)^{2}\right]^{5 / 2}} \times \\
& \times d m_{z \mathrm{Fe}_{3} \mathrm{O}_{4}}\left(x^{\prime}, y^{\prime}, \epsilon\right)+\eta(x, y),
\end{aligned}
$$

onde $\mu_{0}=4 \pi \times 10^{-7} \mathrm{~T} \cdot \mathrm{m} \cdot \mathrm{A}^{-1}$ é a permeabilidade do vácuo. Devido ao fato de que a amostra está perto dos detectores podemos assumir que somente a componente- $z$ do campo magnético é relevante para determinar a res- 
posta do sistema de imagens devido à presença das partículas magnéticas. O último termo, $\eta(x, y)$, é o aporte do ruído durante o processo de registro da imagem, por um sistema linear de imagens magnéticas.

Considerando o sistema de imagens com uma PSF espaço-invariante e o domínio do volume condutor com dimensões dadas no intervalo $\left\{\left(x_{1}, x_{2}\right)\right.$ e $\left.\left(y_{1}, y_{2}\right)\right\}$, o campo magnético total em função da concentração de partículas magnéticas, detectado por um dado sensor magnético é representado pela seguinte equação integral,

$$
\begin{aligned}
& b_{z}(x, y) \\
& =\Lambda \int_{x_{1}}^{x_{2}} \int_{y_{1}}^{y_{2}} d x^{\prime} d y^{\prime} C_{F_{3} O_{4}}\left(x^{\prime}, y^{\prime}\right) \times \\
& \times \frac{2(z-\epsilon)^{2}-\left(x-x^{\prime}\right)^{2}-\left(y-y^{\prime}\right)^{2}}{\left[\left(x-x^{\prime}\right)^{2}+\left(y-y^{\prime}\right)^{2}+(z-\epsilon)^{2}\right]^{5 / 2}} \\
& +\eta(x, y),
\end{aligned}
$$

sendo

$$
\Lambda=\frac{\mu_{0}}{4 \pi}\left(\frac{\mu}{\mu_{0}}-1\right) \frac{\epsilon}{\rho_{F e_{3} O_{4}}} B_{z}^{E X C}
$$

uma função constante, onde $\rho_{\mathrm{Fe}_{3} \mathrm{O}_{4}}$ é a densidade da amostra, $C_{\mathrm{Fe}_{3} \mathrm{O}_{4}}\left(x^{\prime}, y^{\prime}, z^{\prime}\right)$ e $\mu$ são a concentração e a permeabilidade magnética das partículas de $\mathrm{Fe}_{3} \mathrm{O}_{4}$. O campo magnético de excitação que garante a direção de magnetização ao longo do eixo- $z$, é representado por $B_{z}^{E X C}$.

Imagens reais são discretas, consideremos que no processo de medição se faz uma amostragem sobre a amostra numa rede $(n \times$ $m)$ pontos. A imagem magnética discretizada adota a seguinte forma,

$$
\begin{aligned}
& b_{z}\left(x_{n}, y_{m}\right) \\
& =\Lambda \sum_{n=x_{1}}^{x_{2}} \sum_{m=y_{1}}^{y_{2}} C_{F_{3} O_{4} O_{4}}\left(x^{\prime}, y^{\prime}\right) \Delta x^{\prime} \Delta y^{\prime} \times \\
& \times \frac{2(z-\epsilon)^{2}-\left(x_{n}-x_{n^{\prime}}\right)^{2}-\left(y_{m}-y_{m^{\prime}}\right)^{2}}{\left[\left(x_{n}-x_{n^{\prime}}\right)^{2}+\left(y_{m}-y_{m^{\prime}}\right)^{2}+(z-\epsilon)^{2}\right]^{5 / 2}} \\
& +\eta\left(x_{n}, y_{m}\right),
\end{aligned}
$$

a qual pode ser reescrita como o produto de convolução de duas funções

$$
\begin{aligned}
b_{n, m} & \left(x_{n}, y_{m}\right) \\
= & \Lambda \cdot g_{n, n^{\prime}, m, m^{\prime}}\left(x_{n}-x_{n^{\prime}}, y_{m}-y_{m^{\prime}}, z-\epsilon\right) \\
& \otimes C_{F_{3} O_{4}}\left(x_{n^{\prime}}, y_{m^{\prime}}\right) \Delta x_{n^{\prime}} \Delta y_{m^{\prime}} \\
+ & \eta_{n, m}\left(x_{n}, y_{m}\right),
\end{aligned}
$$

ou ainda,

$$
\begin{aligned}
b_{n, m} & \left(x_{n}, y_{m}\right) \\
= & h_{n, n^{\prime}, m, m^{\prime}}\left(x_{n}-x_{n^{\prime}}, y_{m}-y_{m^{\prime}}, z-\epsilon\right) \\
& \otimes C_{F e_{3} O_{4}}\left(x_{n^{\prime}}, y_{m^{\prime}}\right) \Delta x_{n^{\prime}} \Delta y_{m^{\prime}} \\
+ & \eta_{n, m}\left(x_{n}, y_{m}\right),
\end{aligned}
$$

com

$$
\begin{aligned}
& g_{n, n^{\prime}, m, m^{\prime}}\left(x_{n}-x_{n^{\prime}}, y_{m}-y_{m^{\prime}}, z-\epsilon\right) \\
& =\frac{\mu_{0}}{4 \pi} \frac{2(z-\epsilon)^{2}-\left(x_{n}-x_{n^{\prime}}\right)^{2}-\left(y_{m}-y_{m^{\prime}}\right)^{2}}{\left[\left(x_{n}-x_{n^{\prime}}\right)^{2}+\left(y_{m}-y_{m^{\prime}}\right)^{2}+(z-\epsilon)^{2}\right]^{5 / 2}},
\end{aligned}
$$

sendo conhecida a função de Green discretizada [36] e

$$
\begin{aligned}
& h_{n, n^{\prime}, m, m^{\prime}}\left(x_{n}-x_{n^{\prime}}, y_{m}-y_{m^{\prime}}, z-\epsilon\right) \\
& =g_{n, n^{\prime}, m, m^{\prime}}\left(x_{n}-x_{n^{\prime}}, y_{m}-y_{m^{\prime}}, z-\epsilon\right),
\end{aligned}
$$

sendo a PSF do sistema linear de imagens magnéticas.

Do ponto de vista físico a PSF representa a resposta do sistema linear de imagens a qualquer fonte magnética pontual (partículas magnéticas concentradas num ponto da amostra) não importando onde este localizada. A Eq. (12) representa a imagem magnética no espaço real e permite determinar as imagens das fontes magnéticas ou da concentração das partículas de $C_{\mathrm{Fe}_{3} \mathrm{O}_{4}}\left(x_{n^{\prime}}, y_{m^{\prime}}\right)$ dentro do volume condutor usado como objeto amostra. Matematicamente esta imagem é a convolução entre a PSF do sistema e a imagem das fontes magnéticas mais a imagem do ruído presente no processo de detecção da imagem.

Supondo que nosso sistema apresente uma SNR na ordem de $50 \mathrm{~dB}$ o ruído pode ser desconsiderado da Eq. (12) e aplicando nela o teo- 
rema da convolução o qual transforma o produto da convolução de duas funções, no produto de suas correspondentes transformadas de Fourier isto é:

$$
\begin{aligned}
& H\left[K_{x}-K_{x^{\prime}}, K_{y}-K_{y^{\prime}}, z-\epsilon\right] \times \\
& \times C_{\mathrm{Fe}_{3} \mathrm{O}_{4}}\left[K_{x^{\prime}}, K_{y^{\prime}}\right]=B_{z}\left[K_{x}, K_{y}\right],
\end{aligned}
$$

$\mathrm{Na}$ expressão anterior a função $H\left[K_{x}-K_{x^{\prime}}, K_{y}-K_{y^{\prime}}, z-\epsilon\right] \quad$ é a OTF do sistema de imagens magnéticas. Esta função nos mostra como um sinal de uma frequência fixa é propagado através do sistema linear, pelo que o operador degradador pode ser considerado um filtro de frequência, e explicitamente vem dado por:

$$
\begin{aligned}
& H\left(k_{x}, k_{y}, z-\epsilon\right) \\
= & \mathcal{T}\left\{h_{n, n^{\prime}, m, m^{\prime}}\left(x_{n}-x_{n^{\prime}}, y_{m}-y_{m^{\prime}}, z-\epsilon\right)\right\} \times \\
\times & \Delta K_{x^{\prime}} \Delta K_{y^{\prime}},
\end{aligned}
$$

onde $\mathcal{T}$ representa a transformada de Fourier bidimensional rápida e

$$
\Delta K_{x^{\prime}}=\frac{2 \pi}{\Delta x}, \quad \Delta K_{y^{\prime}}=\frac{2 \pi}{\Delta y},
$$

são as distâncias mínimas entre dois pontos nos eixos das frequências espaciais, correspondentes aos eixos $x-y$, respectivamente.

Geralmente a OTF é uma função complexa, portanto apresenta amplitude e argumento ou fase. A função do módulo da OTF é mais usada e é definida como a função de transferência da modulação (MTF das siglas em inglês Modulation Transfer Function). Se a resposta impulsiva do sistema a uma entrada pontual (PSF) é conhecida e de ai suas OTF ou MTF são conhecidas, então é possível calcular a resposta de qualquer entrada arbitraria no sistema linear de imagens magnéticas. Portanto, conhecendo as funções anteriores é possível teoricamente obter as imagens das entradas, em outras palavras a solução do problema magnético inverso [37].

Utilizando a Eq. (13), encontramos a solução do problema magnético inverso via filtros espaciais de Fourier, a qual se reduz a uma simples divisão matemática no domínio da frequência espacial ou de Fourier, da seguinte forma,

$$
=\frac{C_{F_{3} O_{4}}\left[K_{x}, K_{y}, z\right]}{H\left[K_{x}-K_{x^{\prime}}, K_{y}-K_{y^{\prime}}, z-\epsilon\right]},
$$

sendo $H^{-1}\left(k_{x}, k_{y}, z-\epsilon\right)$ o filtro inverso.

Nessa expressão, o filtro inverso é por natureza geralmente filtros passa-baixa ou passaalta. O ruído presente nas medições, assim como o ambiental, pode alterar o processo reconstrução, os filtros-inversos passa-baixa (alta) amplificam o ruído de altas frequências espaciais. A função de Green dentro do filtro no denominador da Eq. (14) provoca que a solução seja dominada pelas instabilidades devido às possíveis singularidades que aparecem pela presença das altas frequências espaciais. Neste casso, o problema inverso torna-se assim instável e deve ser regularizado. Este processo de regularização dos filtros inversos é realizado de um modo direto, usando técnicas de janelas no domínio de Fourier. Aqui mostraremos o uso das janelas de Tukey [37], representada pela função ,

$W_{T}\left(k_{z}, k_{\text {max }}\right)=\left\{\begin{array}{ccc}\left(\frac{1}{2}-\frac{k}{2 k_{\max }}\right) & \text { para } & k<k_{\max } \\ 0 & \text { para } & k>k_{\max }\end{array}\right.$

A função desta janela é atenuar a informação nas regiões do domínio das frequências espaciais onde $H\left(k_{x}, k_{y}, z-\epsilon\right)$ apresente um valor pequeno, portanto a Eq. (14) transforma-se em,

$$
\begin{aligned}
& C_{\mathrm{Fe}_{3} \mathrm{O}_{4}}^{\text {Janelada }}\left(K_{x}, K_{y}, z\right)=W\left(k, k_{\max }\right) \times \\
& \times\left(\frac{1}{H\left(K_{x}-K_{x^{\prime}}, K_{y}-K_{y^{\prime}}, z-\epsilon\right)}\right) \times \\
& \times b_{z}\left(K_{x}, K_{y}, z\right),
\end{aligned}
$$

A qual representaria a imagens das fontes magnéticas reconstruídas no espaço de Fourier. Finalmente, para obter a imagem única da distribuição da magnetização do material que compõe o objeto, dentro da banda limitada das frequências espaciais das medições e da janelafiltro de Tukey, vamos utilizar a transformada 
inversa de Fourier,

$$
C_{\mathrm{Fe}_{3} \mathrm{O}_{4}}\left(x^{\prime}, y^{\prime}\right)=\mathcal{T}^{-1}\left\{C_{\mathrm{Fe}_{3} \mathrm{O}_{4}}^{\text {Janelada }}\left(K_{x^{\prime}}, K_{y^{\prime}}\right)\right\}
$$

O resultado anterior representa a imagem das fontes magnéticas reconstruídas no espaço real.

\section{CONCLUSÕES}

Da revisão desde o ponto de vista experimental constatou-se um aumento do número de trabalhos que utilizaram sistemas multicanais para a detecção de campos magnéticos de origem biológica. Tanto nos estudos preclínicos quanto clínicos mostraram sistemas magnéticos de imagens com resolução espacial que variam na faixa de $(2-5) \mathrm{mm}$. Das análises do formalismo matemático relacionado com a solução do problema magnético direto e inverso no caso de campos magnéticos constantes concluímos que é necessário o conhecimento da função de transferência do sistema ou PSF, assim como algumas informações a priori para a obtenção de uma solução plausível. A idéia é contornar as características destes sistemas de serem malcondicionados, e na prática controlar o nível de ruído (a níveis toleráveis) presente nas imagens e aplicar métodos de filtragem espacial para converter o problema em bem-condicionado e assim encontrar uma versão da solução do PMI.
A solução do problema inverso, usando o método de filtragem espacial de Fourier foi apresentada. Neste método, mostramos o papel fundamental que apresentam as características espectrais das grandezas envolvidas no sistema de imagens, especialmente o processo de filtragem inversa. Contudo, neste trabalho mostramos que existe uma necessidade de um melhor entendimento na colocação prática das técnicas de processamento de reconstrução ou deconvolução das imagens magnéticas para futuras aplicações médicas. $\mathrm{Na}$ atualidade o tema de pesquisas da aplicação das imagens magnéticas na medicina e biologia representa um grande desafio para a comunidade científica contemporânea e exigem setups experimentais que permitam realizar a obtenção de imagens biomagnéticas de elevada resolução espacial da ordem de alguns $\mathrm{mm}$, adquiridas em tempo real. Destacam-se o aumento de sistemas de medição multicanal em relação aos monocanais. Entender e criar ferramentas para a solução do problema magnético inverso em tempo real representa uma das tarefas mais difíceis de serem resolvidas. Novos avanços significariam um grande aporte nas aplicações clinicas da energia eletromagnética, que ajudariam a diagnosticar doenças de grande impacto na saúde do homem.
[1] W. Andrä, H. Danan, W. Kirmsse, H. Kramer, P. Saupe, R. Schmieg, M.E. Bellemann, A novel method for real-time magnetic marker monitoring in the gastrointestinal tract. Phys. Med. Biol. 45, 3081 (2000).

[2] W. Weitschies, O. Kosch, H. Mönnikes, L. Trahms, Magnetic Marker Monitoring: An application of biomagnetic measurement instrumentation and principles for the determination of the gastrointestinal behavior of magnetically marked solid dosage forms. Adv. Drug. Deliv. Rev. 57, 1210 (2005).

[3] E. Stathopoulos, V. Schlageter, B. Meyrat, Y. Ribaupierre, P. Kucera, Magnetic pill tracking: a novel non-invasive tool for investigation of human digestive motility. Neurogastroenterol. Motil. 17, 148 (2005).

[4] D.B. de Araújo, A.A.O. Carneiro, E.R. Moraes, O. Baffa, Biomagnetismo uma nova interface entre a física e a biologia. Ciência Hoje 6, 153 (1999).

[5] O. Baffa, Biomagnetism: Instrumentation and Applications. In: III Escola Brasileira de Magnetismo Jorge André Swieca, 2001. Porto Alegre: UFRGS (2001).

[6] C. Maestú et al. Magnetoencefalografía: una nueva técnica de diagnóstico funcional en neurociência. Rev. Neurol. 28, (11) 1077 (1999).

[7] J. Nenonen et al. Principles of Magnetocardiographic Mapping. Cardiac Mapping. In: M. 
Shenasa, M. Borgreffe, G. Breithardt (orgs.), (2000).

[8] C. Del Gratta, V. Pizzella, F. Tecchio, G.L. Romani, Magneto encephalography - a noninvasive brain imaging method with $1 \mathrm{~ms}$ time resolution. Rep. Prog. Phys. 64, 1759 (2001).

[9] Assessment Program. MEG and MSI: Presurgical Localization of Epileptic Lesions and Presurgical Function Mapping. 18, (6) (2003).

[10] M. Burghoff, J. Nenonen, L. Trahms, T. Katila, Conversion of Magnetocardiographic Recordings between Two Different Multichannel SQUID Devices. IEEE Transactions on Biomedical Engeneering 47, (7) (2000).

[11] Autores? Mathematics and Physics of Emerging Biomedical Imaging. The National Academies Press. (1996).

[12] I. Tavarozzi et al, Magnetocardiography: Current status and perspectives. Part II: Clinical applications. IHJ 1, 151 (2000).

[13] R. Fenicci et al., Multichannel MCG Imaging of Ventricular Preesxcitation in an Unshielded Invase Electrophysiology Laboratory. Biomed Technik 46, 73 (2001).

[14] L.E.F. de los Santos, High-Resolution Imaging Of Magnetic Fields Using Scanning Superconducting Quantum Interference Device (Squid) Microscopy. Dissertation - Faculty of the Graduate School of Vanderbilt University, Nashville (2005).

[15] O. Baffa, R.T. Wakai, P.L. Sousa, R.M.M. Verzola, Fetal Heart Rate Monitored by Magnetocardiogram. Braz. J. Med. Biol. Res. 28, (11-12) 1333 (1995).

[16] O. Baffa, S.A.L. Correa, Magnetic and Electric Characteristics of the Electric Organ of the Electric Fish Gymnotus Carapo. Biophys. J. 63, (2) 591 (1992).

[17] O. Baffa, R.B. Oliveira, J.R.A. Miranda, P.L. Sousa, Mixing Power of Food in the Stomach Evaluated by a Biomagnetic Technique. In: Biomagnetism: Fundamental Research and Clinical Applications - Studies in Applied Electromagnetics and Mechanics. Elsevier Sci. Publ. 753 (1995).

[18] J.F. Borin, O. Baffa, A Low Frequency Current Detector for Biomagnetic Studies. In: T. Yoshimoto, M. Kotani, S. Kuriki, H. Karibe, N. Nakasato (orgs.), Recent Advances in Biomagnetism. Sendai: Tohoku University (1999).

[19] A.C. Bruno, P. Costa Ribeiro, A symmetric Third Order Gradiometer Without External Balacing for Magnetocardiography. Cryogenics 23, 324 (1983).

[20] A.A.O. Carneiro, O. Baffa, R.B. Oliveira,
Study of Stomach Motility Using Magnetic Tracers. Phys. Med. Biol. 44, 1691 (1999).

[21] A.A.O Carneiro, O. Baffa, R.B. Oliveira, C.F.O. Graeff, Construction of a Fluxgate Gradiometric System for the Study of the Magnetic Tracers in the Stomach. In: T. Yoshimoto, M. Kotani, S. Kuriki, H. Karibe, N. Nakasato (orgs.), Recent Advances in Biomagnetism. Sendai: Tohoku University (1999).

[22] Biomagnetism an Interdisciplinary Approach. In: S.J. Williamson, G.L. Romani, L. Kaufman, I. Modena. (orgs.), NATO Advanced Study Institute Series A: Life Sciences, 66. New York: Plenum Press (1983).

[23] W. Weitschies et al., High-Resolution Monitoring of the Gastrointestinal Transit of a Magnetically Marked Capsule. Journal of Pharmaceutical Sciences 86, (11) 1218 (1997).

[24] T. Córdova-Fraga et al., Euterpe Olerácea (Açai) as an Alternative Oral Contrast Agent in MRI of the Gastrointestinal System: Preliminary Results. Magnetic Resonance Imaging 22, 389 (2004).

[25] J.R.A. Miranda, R.B. Oliveira, P.L. Sousa, F.J.H. Braga, O. Baffa, A Novel Biomagnetic Method to Study Gastric Antral Contractions. Phys. Med. Biol. 42, (9) 1791 (1997).

[26] M. Moreira, L.O. Murta, O. Baffa, Imaging Ferromagnetic Tracers with an AC Biosusceptometer. Rev. Sci. Instrum. 71, (6) 2532 (2000).

[27] M.E. Cano, T. Córdova, J.C. Martinez, J.B. Alvarado, M. Sosa. 16-Channel Magnetoresistive Scanner for Magnetic Surface Imaging. Rev. Sci. Instrum. 76, 086106 (2005).

[28] L.A. Corá, F.G. Romeiro, F.C. Paixão, M.F. Américo, R.B. Oliveira, O. Baffa, J.R.A. Miranda, Enteric Coated Magnetic HPMC Capsules Evaluated in Human Gastrointestinal Tract by AC Biosusceptometry. Pharmaceutical Research. 23, (8) (2006).

[29] J.A.L Cruz, A.A.O. Carneiro, L.O Murta Jr., O. Baffa, Imaging Ferromagnetic Tracers with Magnetoresistive Sensors Array. In: Mexican Symposium on Medical Physics, 9 2006. (2006).

[30] J.A.L Cruz, A.A.O. Carneiro, L.O Murta Jr., O. Baffa, Imaging Ferromagnetic Tracers with a Magnetoresistive Sensor Array. In: International Conference on Biomagnetism, 15 2006. Vancouver (2006).

[31] M. Moreira, L.O. Murta, O. Baffa, Imaging Ferromagnetic Tracers with an AC Biosusceptometer. Rev. Sci. Instru. 71, (6) 2532 (2000).

[32] P.C. Hansen, Rank-Deficient and Discrete Ill- 
Posed Problems. SIAM (1998).

[33] J.M. Varah, On the Numerical Solution of IllConditioned Linear Systems with Applications to Ill-Posed Problems. J. Numerical Analysis 10, 257 (1973).

[34] R.J. Hanson. A Numerical Method for Solving FREDHOLM Integral Equations of the First Kind Using Singular Values. J. Numerical Analysis 8, 616 (1971).

[35] J. Hadamard, On the Problems with IllConditional and their Physical Significance. Univ. of Princeton, 49 (1902).

[36] S. Tan, Y. Pei Ma, I.M. Thomas, J.P. Wikswo
Jr. Reconstruction of Two-dimensional Magnetization and Susceptibility Distributions from the Magnetic Field PF Soft Magnetic Materials. IEEE Transactiona on Magnetics, 32, (1) 230 (1996).

[37] R.J. Roth, N.G. Sepulveda, J.P. Wikswo Jr., Using a Magnetometer to Image a Twodimensional Current Distribution. J. Appl. Phys. 65, (1) 361 (1989).

[38] H.C Golub et al., Matrix Computations. Johns Hopkins Press (1989). 